\title{
Planned Study Site Planned Duration
}

National Cancer Institute

\section{Source}

National Cancer Institute. Planned Study Site Planned Duration. NCI Thesaurus. Code C94019.

The intended period of time for the planned study site's participation in the study. 